OPEN ACCESS

Edited by:

Chunhua Zhang,

Algoma University, Canada

Reviewed by:

Seyed Peyman Asadi

University of Mazandaran, Iran

Min Wang,

Central South University Forestry and

Technology, China

*Correspondence:

Mu Zhang

zhangmu@jnu.edu.cn

Specialty section:

This article was submitted to

Health Economics,

a section of the journal

Frontiers in Public Health

Received: 28 June 2021 Accepted: 06 January 2022 Published: 31 January 2022

Citation:

Xu S, Zuo Y, Law R, Zhang M, Han J, Li G and Meng J (2022) Coupling Coordination and Spatiotemporal Dynamic Evolution Between Medical Services and Tourism Development in

Front. Public Health 10:731251 doi: 10.3389/fpubh.2022.731251

\section{Coupling Coordination and Spatiotemporal Dynamic Evolution Between Medical Services and Tourism Development in China}

\author{
Shaogui Xu ${ }^{1,2}$, Yifan Zuo ${ }^{1,2}$, Rob Law ${ }^{3}$, Mu Zhang ${ }^{2 *}$, Jiayu Han ${ }^{2}$, Gaopeng $L^{4}{ }^{4}$ and \\ Juewei Meng ${ }^{5}$ \\ ${ }^{1}$ School of Management, Jinan University, Guangzhou, China, ${ }^{2}$ Shenzhen Tourism College, Jinan University, Shenzhen, \\ China, ${ }^{3}$ Department of Integrated Resort and Tourism Management, Faculty of Business Administration, Asia-Pacific \\ Academy of Economics and Management, University of Macau, Macau, China, ${ }^{4}$ Graduate School of Guangxi Medical \\ University, Nanning, China, ${ }^{5}$ School of Acupuncture and Massage, Guangxi University of Chinese Medicine, Nanning, China
}

This work constructs an evaluation index system and quantitatively explores the coupling coordination relationship between the tourism development system and the medical services system in China. Results show that the degree of coupling coordination between the tourism development system and the medical services system showed a good upward trend in China during the period 2012-2019. However, the relationship was barely balanced, with tourism development lagging. The overall layout shows a spatial pattern of "high in the north and low in the south, high in the east and low in the west." The degree of coupling coordination tends to be randomly distributed from clustered distribution, and the cold-hot spots show a spatial development pattern of "cold in the northwest and hot in the southeast" as time passes. The power of government to regulate has always been an important mechanism affecting the degree of coupling coordination. The study aims to provide reference for the rationalization of medical tourism layout and sustainable development.

Keywords: tourism development, medical services, coupling coordination degree model (CCDM), spatiotemporal dynamic evolution, trend surface analysis, geodetector, China

\section{INTRODUCTION}

From ancient times to the present, a healthy body has been one of the primary demands of human beings at every stage of individual human development. The need for wellness and beauty has become commonplace with the development of society; hence, medical tourism has attracted widespread attention as a growing number of people from high-income countries travel to lowand middle-income countries for healthcare $(1,2)$. Although medical tourism is multi-directional and complex, and no reliable verifiable statistics are available $(3,4)$, relevant information shows a number of patients traveling to developing countries for healthcare, and medical tourism has good prospects for growth $(5,6)$. 
Medical tourism is not a new phenomenon, and it is rapidly growing across the world; however, it has no single definition $(2,4)$. Some scholars consider medical tourism as a subset of health tourism or conflate them $(7,8)$. Meanwhile, most scholars attempt to distinguish medical tourism from health tourism. Connell (9) argues that medical tourism is difficult and often futile to define. Nonetheless, the common characteristics can be distinguished (2). For example, medical tourism mainly refers to tourists who undertake tourism activities across national borders and choose their destinations according to their own intentions, unlike general tourism products $(4,10,11)$. On the basis of these characteristics, this work considers medical tourism as a phenomenon in which travelers cross borders, choose their own destinations, and aim to access medical resources and services (5, 12). Connell (10) argues that international medical tourism has evolved through three stages. Initially, developed countries had advanced medical technology and standards, thereby attracting wealthy medical tourists from other developing countries to travel for medical treatment. In the second stage, many tourists from developed countries were attracted to seek medical treatment in these countries as developing countries improved their medical technology and tapped into traditional medical techniques, coupled with relatively low medical costs. In the third stage, medical tourists from developed and developing countries traveled to each other's countries (10). The international medical tourism market is currently at the third stage.

At present, the demand for medical tourism is rapidly increasing due to aging, sub-health, and rising health-care cost. In the emerging field of medical tourism, many countries see significant economic development potential. Asia-Pacific is the fastest growing and dominant region in the international medical tourism field $(13,14)$. Despite the late start of medical tourism in China, the country is rich in Chinese medicine resources, the national health awareness has increased, and the Chinese medical sector has unique advantages in developing medical tourism with its traditional techniques and the broad market. Moreover, the Chinese government attaches great importance to the development of medical tourism. In 2013, China's State Council proposed to "develop health culture and tourism and encourage regions with the conditions to develop health, sports, and medical tourism for the international and domestic markets" and formally approved the establishment of the Boao Lecheng International Medical Tourism Pilot Zone in Hainan. In 2016, the "Health China 2030" plan was released, and it was proposed to actively promote the integration of health with elderly care, tourism, the Internet, fitness and leisure, and food. However, medical tourism products specifically for foreign tourists are rare, and the number of foreign tourists visiting China to receive medical services is small. In terms of inbound medical tourism, China lags considerably behind Thailand, Singapore, and India $(15,16)$.

Research on medical tourism has become a hot topic. Academics have shown considerable interest in medical services and tourism development. They argue that medical tourism is a "combination of medical services and tourism development" $(6,17)$. Despite a high degree of fit between medical services and tourism development, understanding exactly how they are coupled is a challenging task. Given that the coupling is considerably complex to measure, no scientifically sound evaluation system has been created. Existing studies rarely consider the coupling between the two and neglect to use geographic methods to visualize the spatial characteristics and dynamic evolution of the coupling coordination. Although the medical tourism in China has great potential, research has started late and lacks a mature theoretical framework to guide medical tourism practice $(15,16,18)$. Therefore, an in-depth study of the coupling relationship between medical services and tourism development in China is needed.

This study first establishes the indicator system of medical services and tourism development. Then, a comprehensive level evaluation of medical services and tourism development in 31 provinces of China between 2012 and 2019 was conducted, and the coupling coordination degree model (CCDM) was used to gain an accurate insight into the coupling effect between medical services and tourism development. Finally, the spatial-temporal evolution of the coupled and coordinated relationship between the two systems is analyzed. In summary, this work aims to: (1) reveal the comprehensive development level of medical services and tourism development; (2) analyze the development trend of coupling and coordination between medical services and tourism development through CCDM; (3) examine the spatial and temporal evolution patterns and characteristics of the degree of coupling coordination; and (4) reveal the driving mechanism of the degree of coupling coordination through a geodetector. The study will provide a theoretical basis for the development of medical tourism in China.

\section{LITERATURE REVIEW}

\section{Effect of Tourism Development on Medical Services}

The growth of the tourism industry has stimulated governments to expand investment in medical services. Over the past decade, many countries have identified tourism as a pillar industry that can generate foreign exchange earnings, boost economic growth, increase tax revenues, and create a large number of jobs (19). Tourism spurs the development of sectors, such as retail, hospitality, transport, and infrastructure, and stimulates the growth of local health industries $(20,21)$. Moreover, it will promote host countries' export services, creating good conditions for building a good image of the country and for the development of medical and tourism businesses (1, 20, 22). Accordingly, governments provide a range of subsidies and fiscal incentives to encourage investors and promote the development of healthcare services, such as policies for tax breaks, financial support for equipment, provision of land for healthcare services, and support for overseas healthcare investment (23). In particular, developing countries have attempted to improve the professionalism and maturity of the local healthcare sector by using the latest systems and equipment (24).

Tourism development contributes to the optimization of medical services. In a market where tourism demand tends to be personalized and diversified, people traveling to overseas 


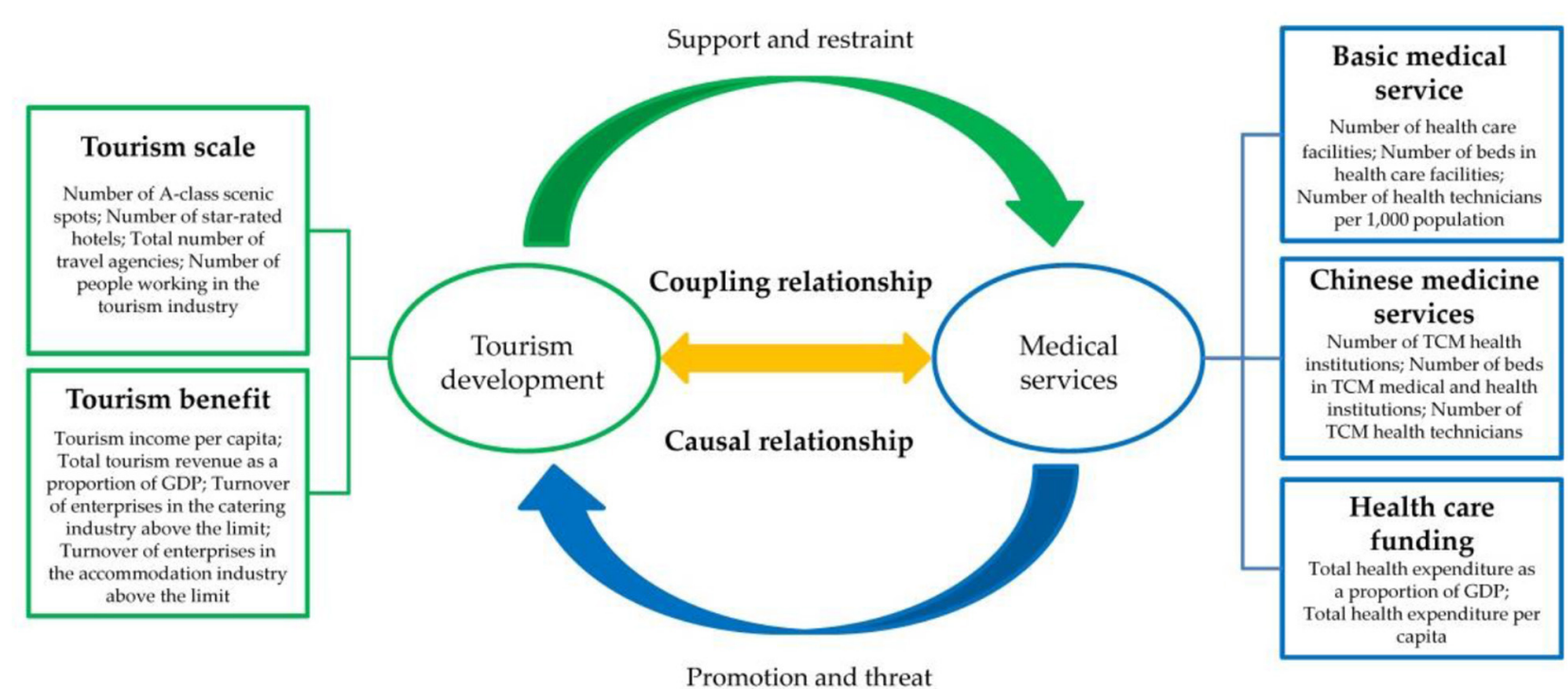

FIGURE 1 | Conceptual framework for the relationship between tourism development and medical services.

TABLE 1 | Coupling coordinated evaluation index system of tourism development and medical services.

\begin{tabular}{|c|c|c|c|c|}
\hline Systems & Subsystems & Indicators & Units & Weight \\
\hline \multirow[t]{8}{*}{ Tourism development } & Tourism scale & Number of A-class scenic spots & Unit & 0.111 \\
\hline & & Number of star-rated hotels & Unit & 0.097 \\
\hline & & Total number of travel agencies & Unit & 0.104 \\
\hline & & Number of people working in the tourism industry & Person & 0.114 \\
\hline & Tourism benefit & Tourism income per capita & Million yuan & 0.095 \\
\hline & & Total tourism revenue as a proportion of GDP & $\%$ & 0.070 \\
\hline & & Turnover of enterprises in the catering industry above the limit & Billion yuan & 0.232 \\
\hline & & Turnover of enterprises in the accommodation industry above the limit & Billion yuan & 0.178 \\
\hline \multirow[t]{8}{*}{ Medical services } & Basic medical service & Number of health care facilities & Unit & 0.156 \\
\hline & & Number of beds in health care facilities & Beds & 0.108 \\
\hline & & Number of health technicians & Thousand person & 0.113 \\
\hline & Chinese medicine services & Number of TCM health institutions & Unit & 0.126 \\
\hline & & Number of beds in TCM medical and health institutions & Beds & 0.101 \\
\hline & & Number of TCM health technicians & Person & 0.116 \\
\hline & Health care funding & Total health expenditure as a proportion of GDP & $\%$ & 0.115 \\
\hline & & Total health expenditure per capita & Yuan & 0.165 \\
\hline
\end{tabular}

countries will want to access medical services, such as dental, cosmetic, fertility, and surgery $(10,25)$. To attract foreign patients, private healthcare providers make significant investments to upgrade their healthcare facilities and train and attract highly skilled healthcare workers (20). Thus, tourism not only contributes to the development of hospitality and transportation but also improves the accessibility and quality of healthcare in many countries (especially developing countries) and can effectively stimulate healthcare providers to provide better quality healthcare services (26), thereby helping in extending the chain and strengthening the link between tourism and healthcare industries (27).
However, tourism development can create inequalities in medical services. The development of tourism has led to significant economic gains and job creation in the health sector for residents of tourist destinations, further increasing the social well-being of residents and improving the quality of life (28). However, the rapid growth of tourism can create conflicts between a large number of tourists and the local population in terms of their healthcare needs. For example, medical tourism could lead to a reduction in access to medical services for the local population, which could have a negative effect on health equity (20). It may lead to the transformation of traditional healthcare services enjoyed by residents into commercial opportunism, such 
as tourist congestion and high costs of healthcare services (9). A growing patron-client conflict between residents and tourists may also ensue (19). Legal and ethical issues may also arise $(29,30)$.

\section{Effect of Medical Services on Tourism Development}

Medical services are encouraging countries to increase investment in tourism. In the broader context of the global healthcare industry, the trends of an aging population, increasing health problems, and rising healthcare expenditure place great pressure on patients $(31,32)$. Accordingly, many patients from developed countries travel abroad to seek low-priced and quality healthcare services (33). Many developing countries are actively investing in the tourism industry, increasing tourism infrastructure, and improving public service support facilities to retain large numbers of tourists and generate objective foreign exchange earnings from tourism (34).

Medical services help increase tourism revenues. The high quality of medical services in a destination country, the effect of branding, and the government's cooperation strategy greatly enhance the attractiveness of a destination, thereby increasing the number of inbound tourists, which, in turn, increases the local tourism revenue $(14,35)$. Stimulated by the huge foreign exchange earnings, the government aims to promote local tourism development by simplifying visa applications, developing transportation, improving tourism infrastructure, and promoting investment $(9,35)$.

However, medical services can affect tourism development. Controversies arise over sensitive medical issues, such as human organ transplants, euthanasia, and abortion, because of the differences in laws and ethics in different countries. From a risk perspective, medical safety incidents are an important influencing factor. For example, when a patient has completed treatment and is likely to travel to another country or return home, continuing treatment or securing legal rights in the event of relapse become difficult, which can increase a patient's medical costs $(36,37)$.

\section{Relationship Between Tourism Development and Medical Services}

The relationship between medical services and tourism development is complex because they have their structures, functions, and development rules and are also constrained by each other (38). The development of tourism provides a medium for the promotion of medical services, and the improvement of the level of medical services causes a tourism product to become diversified and better suited to the needs of tourists. A positive relationship (or negative relationship) can be observed between the two systems (39). The degree of coupling refers to the strength of the interaction between the two parties, regardless of the benefits and drawbacks. The degree of coordination refers to the magnitude of benign coupling in the interaction, reflecting a good or bad coordination status, and can characterize whether the functions promote each other at a high level or constrain each other at a low level $(40,41)$. Coupled coordination analysis refers to the quantitative analysis of the degree of association between two or more systems and can be used to measure the tightness of the relationship between systems $(42,43)$.

At present, researchers have used this approach to study the relationship between tourism and economy $(44,45)$, tourism and culture (46), tourism and environment $(43,47)$, urbanization and environment (48), economic development and ecology (49), and urbanization and geo-hazards (39). These related studies reveal the correlated roles between two systems and indicate that they dynamically change over time (50). Although coupled coordination has been widely applied in the tourism sector, no studies using this method have explored the interactions between medical services and tourism development. Therefore, this study focuses on the spatial-temporal correlation and heterogeneity between the development of medical services and tourism in China to fill the gaps in existing research. The possible causes of conflicts between medical services and tourism development can be identified by analyzing their degree of coupling and coordination, which will help in the promotion of their coordinated development and contribute to the sustainable development of medical tourism.

\section{MATERIALS AND METHODOLOGY}

\section{Indicator System}

An extremely complex interaction exists in the relationship between medical services and tourism development. Tourism development promotes the growth of medical services, whereas medical services support the tourism development. These two aspects complement each other in a dynamic process. Tourism development has a huge tourism multiplier effect on medical services, which not only brings major economic benefits and creates employment opportunities but also drives the development of upstream and downstream industries and extend the medical services industry chain $(20,21)$. However, it can also create social problems, such as inequality in healthcare services (20). Correspondingly, medical services also greatly support the development of the tourism industry.

Medical services provide professional technical support to the tourism industry, enriching the range of tourism products, improving the quality of services, and promoting the upgrade and optimization of the tourism industry structure $(9,14,35)$. However, the health services sector can also have an influence on tourism $(36,37)$. Accordingly, the two aspects are causally related, interdependent, and indispensable. Given that tourism is an integrated industry, it is closely related to other industries and social services (44). Therefore, a system consisting of a tourism development system and a healthcare service system can be defined as a coupled and coordinated system, and the coordination of the coupling is the basis for achieving sustainable development of medical tourism (Figure 1).

We constructed a comprehensive evaluation indicator system using existing indicators to measure the close relationship between tourism development and medical services. The selection of indicators was based on these principles: first, the most frequently cited indicators were chosen; second, the components of tourism development and healthcare services 
TABLE 2 | Classification standard of coupling coordination types between tourism development (TD) and medical services (MS).

\begin{tabular}{llll}
\hline CCD & Stages of coupling development & Relationship between $\mathbf{q}_{\mathbf{1}}$ and $\mathbf{q}_{\mathbf{2}}$ & Development modes between systems \\
\hline $0.8<D \leq 1$ & Superiorly balanced development & $q_{1}>q_{2}$ & $\begin{array}{l}\text { Superiorly balanced development with MS lagged } \\
\text { Superiorly balanced development between TD and MS } \\
\text { Superiorly balanced development with TD lagged }\end{array}$ \\
& & $q_{1}=q_{2}$ & Favorably balanced development with MS lagged \\
$0.6<D \leq 0.8$ & $q_{1}<q_{2}$ & Favorably balanced development between TD and MS \\
& & $q_{1}>q_{2}$ & Favorably balanced development with TD lagged \\
$0.5<D \leq 0.6$ & $q_{1}=q_{2}$ & Barely balanced development with MS lagged \\
& Barely balanced development & $q_{1}<q_{2}$ & Barely balanced development between TD and MS \\
& & $q_{1}>q_{2}$ & Barely balanced development with TD lagged \\
$0.4<D \leq 0.5$ & Slightly imbalanced development & $q_{1}=q_{2}$ & Slightly imbalanced development with MS lagged \\
& & $q_{1}<q_{2}$ & Slightly imbalanced development between TD and MS \\
& & $q_{1}>q_{2}$ & Slightly imbalanced development with TD lagged \\
$0.2<D \leq 0.4$ & Moderately imbalanced development & $q_{1}=q_{2}$ & Moderately imbalanced development with MS lagged \\
& & $q_{1}<q_{2}$ & Moderately imbalanced development between TD and MS \\
& & $q_{1}>q_{2}$ & Moderately imbalanced development with TD lagged \\
$0<D \leq 0.2$ & Seriously imbalanced development & $q_{1}=q_{2}$ & Seriously imbalanced development with MS lagged \\
& & $q_{1}<q_{2}$ & Seriously imbalanced development between TD and MS \\
& & $q_{1}>q_{2}$ & Seriously imbalanced development with TD lagged
\end{tabular}

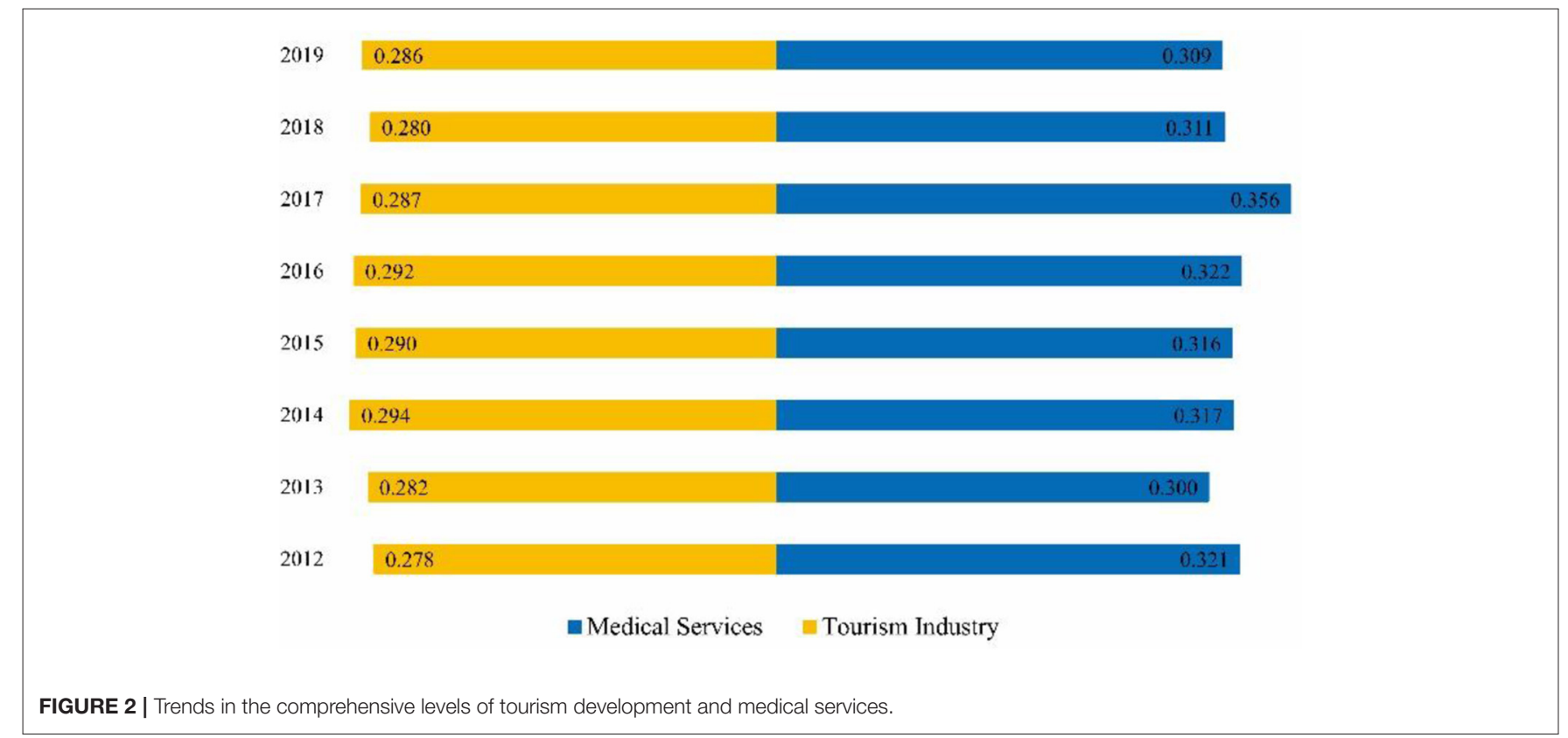

were included; and third, the indicators chosen were of good typicality and practicality. Indicators for tourism development systems were initially identified according to previous studies $(43,44,51,52)$. Indicators for the healthcare delivery system were then further selected through analysis of relevant literature and in the context of China-specific situations to gain insight into the coupling between tourism development and healthcare delivery and identify the interactions between these factors. Finally, a comprehensive evaluation indicator system, consisting of 2 systems, 5 subsystems, and 16 indicators, was constructed (Table 1).
According to industrial development theory, the tourism development system is a complex system whose industrial development is driven by a variety of factors, and the tourism development system evaluation index system can be examined from two subsystems $(43,44,53)$. The first subsystem is the scale of tourism. It reflects the overall level of the tourism industry. The number of A-class scenic spots is used to reflect the endowment value and supporting capacity of tourism resources $(51,54)$. The number of star-rated hotels reflects the overall level of the tourism industry $(54,55)$. The total number of travel agencies reflects the service capacity of the tourism industry $(53,56)$. 
TABLE 3 | Coupling coordination degree and the development stage between tourism development and medical services.

\begin{tabular}{|c|c|c|c|c|}
\hline Year & C & $\mathbf{T}$ & D & Coupled and coordinated development phase \\
\hline 2012 & 0.945 & 0.300 & 0.520 & Barely balanced development with TD lagged \\
\hline 2013 & 0.952 & 0.291 & 0.513 & Barely balanced development with TD lagged \\
\hline 2014 & 0.950 & 0.306 & 0.526 & Barely balanced development with TD lagged \\
\hline 2015 & 0.954 & 0.303 & 0.525 & Barely balanced development with TD lagged \\
\hline 2016 & 0.953 & 0.307 & 0.529 & Barely balanced development with TD lagged \\
\hline 2017 & 0.948 & 0.322 & 0.539 & Barely balanced development with TD lagged \\
\hline 2018 & 0.954 & 0.296 & 0.518 & Barely balanced development with TD lagged \\
\hline 2019 & 0.961 & 0.297 & 0.520 & Barely balanced development with TD lagged \\
\hline
\end{tabular}

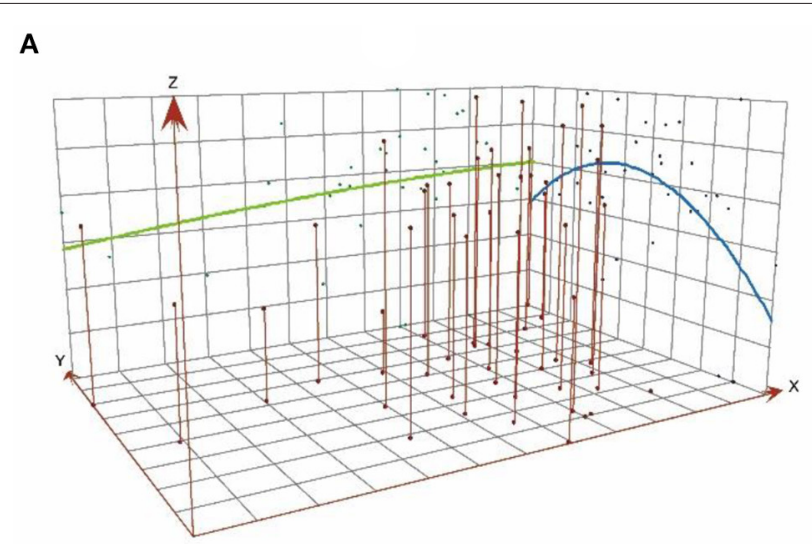

B

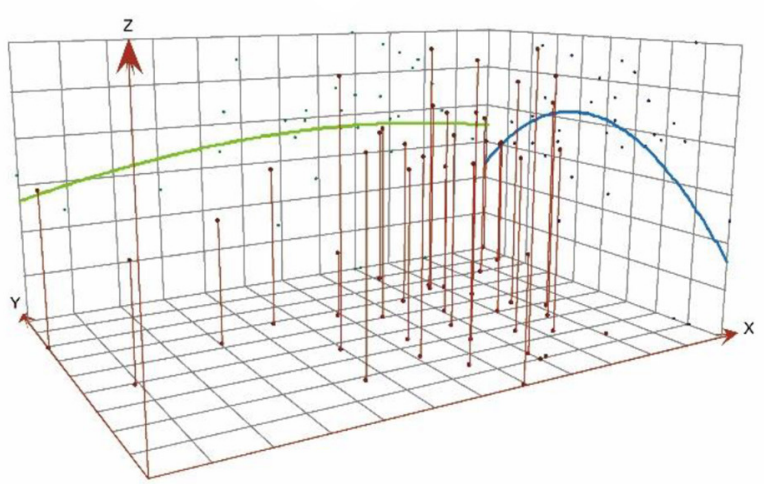

FIGURE 3 | Analysis of spatial trends in the coupled coordination of tourism development and medical services (A) 2012, (B) 2019.

Moreover, the number of people working in the tourism industry reflects the tourism labor inputs $(53,57)$. The second subsystem is tourism benefit, which reflects the economic benefits and status of the tourism industry. The tourism income per capita reflects the economic effect of tourism $(43,51)$. The total tourism revenue as a proportion of GDP reflects the position of tourism in the national economy (53). The turnover of enterprises in the catering industry above the limit reflects the share of the catering sector in the tourism revenue structure (52). Moreover, the turnover of enterprises in the accommodation industry above the limit reflects the share of the accommodation sector in the tourism revenue structure $(43,52)$.

China's healthcare delivery system operates on a dual-track basis, including the public and private sectors. Public healthcare institutions are the mainstay and cornerstone of the healthcare delivery system (58). Chinese medicine and Western medicine co-exist in the current health care delivery system. Accordingly, this study considers three subsystems of the healthcare delivery system. The first subsystem is primary care services. The content of primary healthcare services mainly includes various institutions and facilities of disease treatment, convalescence and recuperation, treatment and examination, and the corresponding consumption of medicines. Thus, we measure the conditions for the development of basic medical services in terms of institutions, facilities, and personnel, which mainly include the number of health care facilities (24), the number of beds in health care facilities (14), and the number of health technicians $(59,60)$. The second subsystem is Chinese medicine services. Traditional Chinese medicine (TCM) not only plays an important role in the Chinese healthcare system, but it is also welcomed and respected worldwide in promoting health, well-being, and longevity. In particular, TCM has significantly contributed to the treatment of COVID-19 $(61,62)$. Chinese medicine services also measure the conditions for the development of Chinese medicine services in terms of institutions, facilities, and personnel, including the number of TCM health institutions (62), number of beds in health care facilities (61), and the number of TCM health technicians $(58,63)$. The third subsystem is health care funding, which mainly reflects the government's financial investment in health care services and the extent to which the government and society as a whole attach importance to the health of the population. Therefore, the evaluation indicators of health care funding include total health expenditure as a proportion of GDP $(20,60)$ and the total health expenditure per capita (64).

\section{Data Collection}

The latest data available for this study are from 2019 because of the lag in the publication of statistics in China. Accordingly, we choose the period 2012-2019 as the study period and took 31 provinces (cities and districts) in China (excluding Hong Kong, Macao, and Taiwan) as the research object. The data used are mainly from the China Statistical Yearbook, the China Health 
TABLE 4 | Global autocorrelation analysis of the coupling coordination degree of tourism development and medical services.

\begin{tabular}{lcccl}
\hline Year & Moran's $\boldsymbol{I}$ & $\boldsymbol{Z}$-value & $\boldsymbol{p}$-value & Spatial pattern \\
\hline 2012 & 0.120 & 1.999 & 0.046 & Clustered distribution \\
2013 & 0.128 & 2.104 & 0.035 & Clustered distribution \\
2014 & 0.121 & 2.014 & 0.044 & Clustered distribution \\
2015 & 0.108 & 1.849 & 0.045 & Clustered distribution \\
2016 & 0.132 & 2.162 & 0.031 & Clustered distribution \\
2017 & 0.073 & 1.383 & 0.167 & Random distribution \\
2018 & 0.089 & 1.583 & 0.114 & Random distribution \\
2019 & 0.074 & 1.383 & 0.167 & Random distribution
\end{tabular}

Care Statistical Yearbook, and the statistical bulletin on the national economic and social development of each province.

\section{Methods}

\section{Integrated Development Level Evaluation Model (IDLEM)}

The medical service system and the tourism development system are two systems that influence each other, and the level of their combined development needs to be measured. The calculation formula is as follows:

$$
u_{i}=\sum_{j=1}^{m} \lambda_{i j} u_{i j}, \sum_{j=1}^{m} \lambda_{i j}=1
$$

where $u_{i}$ refers to the comprehensive evaluation value of the system in year $i, u_{i j}$ is the degree of contribution of indicator $j$ to the system, and $\lambda_{i j}$ refers to the weight of the indicator. The degree of contribution $u_{i j}$ is calculated according to the effect of the indicator on the system using a positive efficacy function, as follows: $u_{i j}=\left(X_{i j}-X_{j \min }\right) /\left(X_{j \max }-X_{j \min }\right)$, where $X_{j \max }$ and $X_{j \min }$ are the maximum and minimum values of the $j$ th indicator, respectively. The indicator weights $\lambda_{i j}$ are calculated by using the entropy method, which is objective and scientific. The entropy method can judge the degree of dispersion of indicators based on the size of information provided by the observations of each indicator and assign the indicator weights (43). First, the data must be standardized or normalized. The standardization of positive and negative indicators is distinguished because the magnitudes of the data used are different; second, the entropy value of each indicator is calculated; again, the weights of each indicator are determined according to the entropy value of each indicator (65-67). The entropy method is general, and the calculation steps are not repeated $(68,69)$. Table 1 shows the weights of each indicator.

\section{CCDM}

The coupling equation in physics is used to model the degree of coupling between the medical services system and the tourism development system:

$$
C=2 \sqrt{\frac{q_{1} \times q_{2}}{\left(q_{1}+q_{2}\right)^{2}}}
$$

where $C$ is the degree of coupling of the two systems. Relying solely on the degree of coupling is not possible because the coupling model can give the illusion that the degree of coupling is high when the comprehensive evaluation index of the system is low. Therefore, the coupling coordination model is constructed as follows:

$$
\begin{array}{r}
D=\sqrt{C T} \\
T=\alpha q_{1}+\beta q_{2}
\end{array}
$$

where $D$ is the coupling coordination degree; $T$ is the comprehensive evaluation index; and $\alpha$ and $\beta$ are the weights of the medical services system and the tourism development system, respectively. Weights $\alpha$ and $\beta$ are assigned to 0.5 by referring to related studies $(40,70)$; the higher the value of the degree of coupling coordination, the better the match between the two systems, and the system tends to develop in an orderly direction. In addition, the degree of coupling coordination and the comprehensive development level were ranked according to related studies to determine their development stages $(39,69)$ (Table 2).

\section{Trend Surface Analysis}

Trend surface is a semi-quantitative study of geographic data with a large spatial span based on spatial data and mathematical surfaces emulated by mathematical fitting, which can be used to explore the spatial trends and distribution patterns of geographic element observations $(71,72)$. In this work, we simulate the spatial and temporal variation characteristics of the coupling coordination status of China's medical services system and tourism development system in 2012 and 2019 by using trend surface analysis and the degree of coupling coordination as the observation. Let $\left(X_{i}, Y_{i}\right)$ be the spatial location of the $i$ th province and $Z_{i}\left(X_{i}, Y_{i}\right)$ be the trend function of the $i$ th province, where the $X$-axis represents the east-west direction, and the $Y$-axis denotes the north-south direction.

\section{Spatial Autocorrelation}

Spatial autocorrelation is a description of the degree of similarity and spatial association between the attribute values of spatially adjacent regional units and is used to reveal the spatial interactions of a geographical phenomenon between contiguous territories $(73,74)$.

In this work, global spatial correlation is measured using Moran's $I$, which takes the values in the range $[-1,1]$. The closer Moran's $I$ is to 1 , the stronger the degree of agglomeration, and the closer it is to -1 , the stronger the degree of dispersion. An index of $<0$ indicates a negative correlation; and that $>0$ denotes a positive correlation. A random distribution is signified as it equals to 0 ; and a result suggesting 1 or -1 demonstrates an extremely strong positive or negative correlation (74). The specific formula for the global Moran's I is as follows (39): 


$$
I=\frac{n \sum_{i=1}^{n} \sum_{j=1}^{n} \omega_{i, j}\left(x_{i}-\bar{x}\right)\left(x_{j}-\bar{x}\right)}{\sum_{i=1}^{n} \sum_{j=1}^{n} \omega_{i, j} \sum_{i=1}^{n}\left(x_{i}-\bar{x}\right)^{2}}
$$

where $n$ is the number of observations of variable $x ; x_{i}$ and $x_{j}$ are the observations of variable $x$ at positions $i$ and $j$, respectively; $\bar{x}$ is the mean of all observations; and $\omega_{i, j}$ is the spatial weight matrix value.

We also identify high- and low-value agglomerations of the coupled coordinated development across provinces, that is, hot and cold spots to observe local characteristics, on the basis of the Getis-Ord $\mathrm{Gi}^{*}$ statistic in ArcGIS to avoid global spatial autocorrelation that may mask local spatial heterogeneity (75).

\section{Geodetector}

Geodetector is a set of statistical methods that detect spatial variability and reveal the driving forces behind it. It can detect the extent to which a factor explains the spatial variation of the dependent variable and reveal the source of spatial variation in the dependent variable (76). Moreover, it is widely used in nature and society because it does not require the assumption of linearity, and it has become a master method in geography to detect the mechanisms of regionalism and variability. The model expresses as follows:

$$
q=1-\frac{1}{N \sigma^{2}} \sum_{h=1}^{L} N_{h} \sigma_{h}^{2}
$$

where $h$ is the stratification of the variable or factor; $L$ is the number of stratification of the variable or factor; $N$ and $N_{h}$ are the number of cells of the full region and stratification $h$, respectively; $\sigma^{2}$ and $\sigma_{h}^{2}$ are the variance of the full region and stratification $h$, respectively.

The value of $q$ is used to measure the degree of explanation of the spatial variance of the detection factor $X$ on the attribute $Y$. The value of $q \in[0,1]$. A larger $\boldsymbol{q}$ value indicates a greater influence of the detection factor on the variable, and the stronger the heterogeneity. When $\boldsymbol{q}=0$, the detection factor has the weakest explanatory power on the variables, and there is no spatial heterogeneity; when $\boldsymbol{q}=1$, the detection factor has the greatest explanatory power on the variables and has high spatial heterogeneity.

\section{RESULTS}

\section{Results of the Comprehensive Levels}

A comprehensive level index of China's tourism development system and medical services system from 2012 to 2019 was calculated using Equation (1) (Figure 2). Overall, the development level of tourism development and medical services in China showed a slight upward trend in fluctuation. The results of the tourism development comprehensive measure indicate that the development level index of tourism development increased from 0.278 in 2012 to 0.286 in 2019. Meanwhile, the development level index was $>0.290$ from 2014 to 2016, with an average annual growth rate of $3.1 \%$. This result indicates that
China's tourism industry has undergone a slow upward trend during this period.

The results of the comprehensive measure of medical services demonstrated that the development level index increased from 0.309 in 2012 to 0.321 in 2019. In particular, the average annual growth rate of $9.1 \%$ for 2013-2017 indicates that Chinas medical services industry has developed at a fast rate during this period. The comparison of the index of tourism development and medical services indicated that the development trend of tourism development and medical services in China was the same from 2012 to 2019. However, differences were also observed. The level of medical services development becomes much better than tourism development as the gap between the index of tourism development and medical services increases. During these 5 years, the income of residents has increased as China has built a moderately prosperous society in an all-round way, and the paid leave system has been gradually implemented. China's tourism has shifted from the development model of attraction tourism to the development model of holistic tourism (40). However, the pace of tourism development has slowed down compared with that of the previous decade. China is the world's most populous country, and its large population base and rapidly growing aging population bring about a continuously growing demand for healthcare services; accordingly, the healthcare service industry is developing at a faster rate (30). Therefore, some differences can be observed between the level of medical service and tourism development.

\section{Results of the Degree of Coupling Coordination}

The degree of coupling coordination of the tourism development system and medical services system from 2012 to 2019 was calculated on the basis of Equations (2)-(4). Then, the coordination development stages were divided (Table 3). During this period, the degree of coupling slightly changed, and the average value is $>0.9$. The change in the degree of coordination and the coupling coordination is not significant. The above data show that although the overall coupling and coordination between tourism development and medical services show a slight increasing trend, it is only at the stage of barely balanced development. Moreover, the level of coupling coordination between the two aspects requires further improvement. According to the comparison of the comprehensive development level, coordination and interaction can be divided into three types. If $q_{1}>q_{2}$, then medical services are lagging; if $q_{1}>q_{2}$, then tourism development and medical services are simultaneously developing; if $q_{1}<q_{2}$, then tourism development is lagging. Table 3 shows that tourism development was always lagging behind from 2012 to 2019 .

A spatial trend analysis was conducted on the basis of ESDA technology to further reveal the state of coupling coordination between tourism development and medical services in different regions of China. Figure 3 shows the results. The overall spatial pattern of the degree of coupling coordination is "high in the north and low in the south, high in the east and low in the west." In the figure, the $X$-axis is the east direction, the $Y$-axis 
A

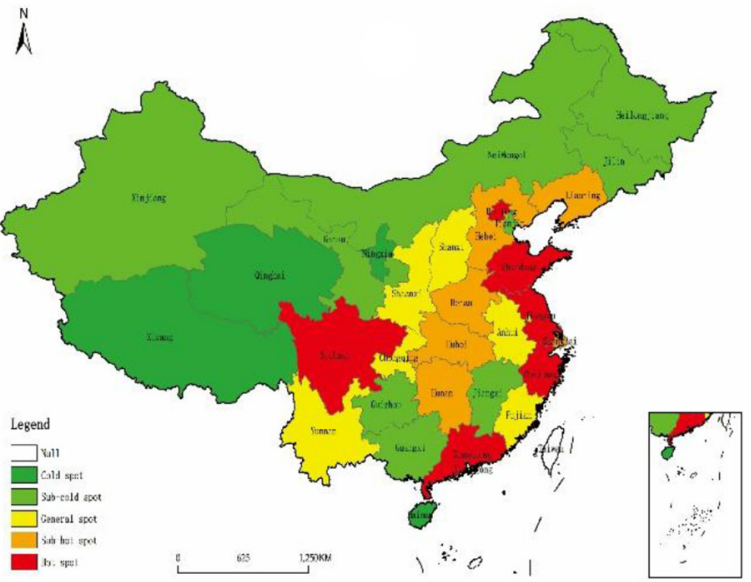

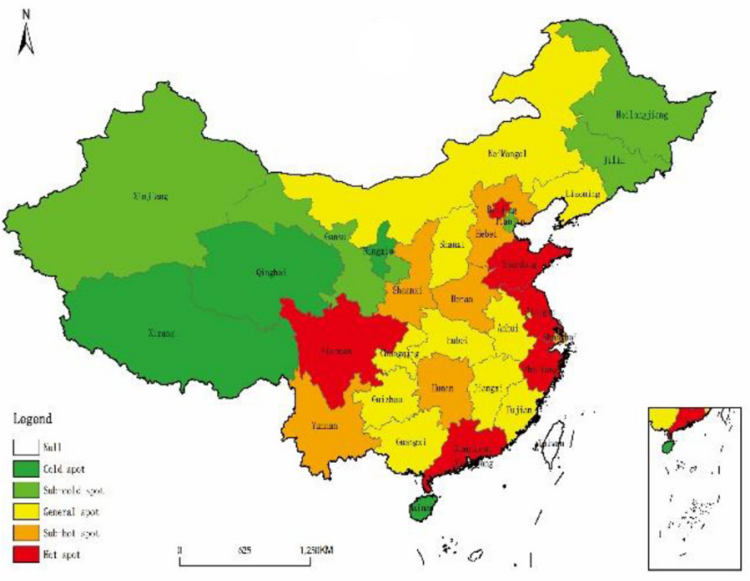

FIGURE 4 | Cold-hot spots of the degree of coupling coordination of tourism development and medical services (A) 2012 , (B) 2019.

TABLE 5 | Driving factors affecting coupling and coordination between tourism development and medical services ( $q$ value).

\begin{tabular}{|c|c|c|c|c|c|c|c|c|c|c|}
\hline Driving mechanism & Detection factors & 2012 & 2013 & 2014 & 2015 & 2016 & 2017 & 2018 & 2019 & Mean \\
\hline \multirow[t]{2}{*}{ Economic development level } & Per capita GDP (yuan) & 0.290 & 0.292 & 0.269 & 0.255 & 0.288 & 0.250 & 0.268 & 0.261 & 0.272 \\
\hline & Per capita disposable income (yuan) & 0.348 & 0.354 & 0.354 & 0.311 & 0.384 & 0.214 & 0.331 & 0.277 & 0.322 \\
\hline Industry structure & Proportion of tertiary industry (\%) & 0.099 & 0.086 & 0.090 & 0.334 & 0.282 & 0.073 & 0.166 & 0.263 & 0.174 \\
\hline \multirow[t]{2}{*}{ Transportation conditions } & Total length of highways (km) & 0.279 & 0.279 & 0.177 & 0.246 & 0.178 & 0.243 & 0.168 & 0.195 & 0.221 \\
\hline & Length of railways in operation (km) & 0.133 & 0.140 & 0.111 & 0.103 & 0.108 & 0.157 & 0.081 & 0.105 & 0.117 \\
\hline \multirow[t]{2}{*}{ Ecology } & Forest coverage rate (\%) & 0.126 & 0.125 & 0.128 & 0.124 & 0.137 & 0.166 & 0.097 & 0.150 & 0.132 \\
\hline & Per capita area of park green land $\left(m^{2}\right)$ & 0.032 & 0.075 & 0.129 & 0.086 & 0.104 & 0.081 & 0.146 & 0.330 & 0.123 \\
\hline Urbanization level & Proportion of urban population (\%) & 0.310 & 0.304 & 0.215 & 0.244 & 0.280 & 0.151 & 0.198 & 0.218 & 0.240 \\
\hline \multirow[t]{2}{*}{ Government regulation ability } & Per capita budgetary expenditure (yuan/per person) & 0.254 & 0.280 & 0.262 & 0.137 & 0.319 & 0.363 & 0.363 & 0.345 & 0.290 \\
\hline & Total investment in fixed assets (100 million yuan) & 0.734 & 0.729 & 0.723 & 0.698 & 0.680 & 0.599 & 0.645 & 0.221 & 0.629 \\
\hline
\end{tabular}

is the north direction, and the $Z$-axis represents the degree of coupling coordination of each province. The blue curve is the best-fit curve of the coupling coordination degree in the northsouth direction, and the green curve is the best-fit curve of the coupling coordination degree in the east-west direction.

From the characteristics of the curve change, the northsouth direction shows a clear spatial distribution of "high in the middle and low on both sides, and high in the north and low in the south." The provinces with high values of coupling coordination include Shandong, Beijing, Zhejiang, Jiangsu, and Shanghai, most of which are located in the coastal area in the north of China. Beijing is unique because it is the national capital. The other provinces are located near the coast. Their high level of development of medical services and tourism and their high socio-economic conditions provide a good basis for the coupling and coordination compared with the inland. By contrast, the low-value coupling coordination zone is located mainly in the provinces of Anhui, Yunnan, Guangxi, Fujian, and Hainan, most of which are located in the south of China. From the socio-economic point of view, these provinces are much less developed than the northern coastal areas, thus having a lower degree of coupling coordination. The feature in the north-south direction remains stable over time and has not significantly changed. The trend in the eastwest direction is more active, evolving from a "high in the east and low in the west" to an inverted " $U$ " shape. However, the curve always maintains the characteristic in which the east is higher than the west. The gap between the east and the west ends is slightly moderated, indicating that the gap between the coupling of tourism development and medical services in China's provinces is gradually decreasing in the east-west direction, and the inter-regional coordination improved to a certain extent.

The reasons for this result are inseparable from the geographical location and economic and social conditions. In the east-west direction, the provinces of Ningxia, Tibet, and Qinghai in the western region of China have a harsh ecological environment, making it difficult to develop tourism (38). The regions located in the inland northwest have a low level of economic development and inadequate health care infrastructure. By contrast, the eastern provinces have convenient transportation, pleasant climate, and many international cities 
with relatively high socioeconomic and cultural levels, providing a good foundation for medical services and tourism development (51). Although the north also has a certain tourism base and a high level of urbanization, the medical environment is not comfortable compared with the south. The south has a warm and humid climate with high forest vegetation cover and a sound healthcare infrastructure. Thus, the overall level of development coordination is higher than that in the north.

\section{Spatial Pattern and Development Characteristics}

Moran's I and its related indicators for the coupling coordination degree of tourism development and medical services in China from 2012 to 2019 were calculated by using Equation (5) to test the geospatial correlation of the coupling coordination degree, as shown in Table 4. The results show that Moran's $I$ was positive in the period 2012-2016, ranging from 0.120 to 0.132 , and it passed the $5 \%$ significance test level (77). This notion indicates that there is a significant spatial clustering trend in the coupling coordination degree over this period, with a "proximity dependency" effect between geographically adjacent provinces. The Moran's $I$ and Zvalue gradually decreased with $p>0.05$ between 2017 and 2019. The spatial pattern of the coupling coordination degree transformed from clustered distribution to random distribution. The overall development level of tourism development and medical services in China was improved, and the development was balanced.

The Getis-Ord Gi* statistics was used to map the spatial and temporal distribution of cold-hot spots in the coupling coordination degree of tourism development and medical services in China in 2012 and 2019 and further explore the local spatial pattern (Figure 4). The hot and cold spot areas do not greatly change. By contrast, the sub-hot, subcold, and general areas evidently change. This result indicates an overall spatial development pattern of "cold in the northwest and hot in the southeast," with an obvious gradient distribution pattern.

The hot spot areas are concentrated, with the radiation range roughly showing a strip-like distribution along the eastern coast. The cold spot areas are scattered but concentrated in the west with a clump-like distribution. The sub-hot spot areas distributed from a strip pattern extending from the center to the north to a sporadic point pattern. The general area shows a group-loading distribution and has increased by four provinces from 2012 to 2019. The spatial pattern of the sub-hotspot area shows a striplike distribution in the north and a group-packed distribution in the south. Then, the pattern shows a spatial distribution trend of decreasing in the north and expanding in the south. These results show significant regional differences in the degree of coupling coordination of tourism development and medical services in China. The regions with a high overall level of coordinated development are concentrated mainly in provinces with good health care conditions or developed tourism industry. Furthermore, there may be a link between this distribution characteristic and the degree of economic development of each province.

\section{Driving Mechanism of Coupling Coordination}

The coordinated development of tourism development and medical services is a more complex process driven by a variety of factors, such as natural, economic, policy, and accessibility. To verify the results of the previous analysis and considering the availability of data, the factors, such as economic development level, industry structure, transportation conditions, ecology, urbanization level, and government regulation ability, can be combined with the results of other scholars' studies. Accordingly, an aggregated index system consisting of six aspects and 10 detection factors was formulated based on literature and considering the data availability (Table 5) (27, 38, 41, 53, 78). ArcGIS software was used to classify the natural break points of each factor, and the driving mechanism of coupling coordination between tourism development and medical services was detected and analyzed according to the geodetector.

According to Table 5, the differences in the influence of each factor of coupling coordination of tourism development and medical services from 2012 to 2019 are more obvious. First, the power of the government to regulate is always an important factor influencing the coordinated development. In particular, total investment in fixed assets has the greatest influence with its mean value of 0.629 , but its influence decreases year by year. Then, the level of economic development and urbanization also have a large influence. Other factors also have different degrees of influence on the degree of coordination in different years. The specific influencing mechanisms are as follows.

(1) Economic development level. The $q$-values of per capita GDP and per capita disposable income are $>0.2$, indicating that economic development level has a strong driving effect on the integration of tourism development and medical services. The large amount of capital investment helps the infrastructure construction of tourism and medical services development and strengthens the competitive advantage of the industry. The overall consumption level of residents also rises, which is conducive to promoting the scale of tourism and medical services demand, helping tourism and medical services and other industrial factors to achieve the agglomeration effect.

(2) Industrial structure. The $q$-value of proportion of tertiary industry varies from 0.073 to 0.334 . Given that tourism and medical services belong to the category of tertiary industry, the general environment of the development of tertiary industry has a certain influence on their integration and development.

(3) Transportation conditions. According to the results of the geodetector analysis, the mean values of $q$-value of the total length of highways and railways in operation are 0.221 and 0.117 , respectively. Overall, transportation is the most important factor affecting the coordination development of tourism and medical services. However, the total length of highways has a greater effect. From the overall situation in China, the railways as the external transportation facilities between regions have been relatively well-developed, but the unbalanced between regions. 
Therefore, it is the basic condition that affects the coordination development of tourism development and medical services.

(4) Ecology. The $q$-value of forest coverage rate shows a small continuous increase, and the per capita area of park green land is fluctuating in an upward trend. This result indicates that the sustainability of the ecological environment of the whole region is more important for the coordinated development of tourism development and medical services than the urban environment.

(5) Urbanization level. The mean $q$-value for the proportion of urban population is $>0.2$, indicating that the level of urbanization plays a significant role in the development of tourism and medical services, and it is a prerequisite for their coupled and coordinated development. The higher the level of urbanization, the stronger the service industry's reception capacity.

(6) Government regulation ability. Government regulation is an important manifestation of government action on tourism development and medical services development, and it is an external guarantee force to promote the coordinated development of both. Table 5 demonstrates that the influencing value of per capita budgetary expenditure increases year by year, and the average value of $q$ for total investment in fixed assets is $>0.6$, indicating that the government regulation ability is increasingly important to promote the coordinated development of tourism development and medical services.

\section{DISCUSSION}

\section{Temporal Dynamic Changes}

The coupled and coordinated development of tourism development and medical services is a dynamic process. The coordinated development of the two aspects has an important role in promoting the sustainable development of medical tourism. The study results show that the trends in the overall development of tourism development and medical services in China are consistent, and both are on the rise. However, the gap between the two aspects is increasing, with medical services developing at a faster rate than tourism. Moreover, the coupling of tourism development and medical services systems is only at the barely balanced development stage with tourism lagging behind. This finding suggests that the development of tourism development and medical services in China is uneven. China is the world's most populous country, and its large population base and rapidly growing aging population have created a growing demand for medical services (62). The healthcare service industry is not only an important part of the healthcare sector but also a new growth area for the modern service industry because it is closely related to national strategies (30). Accordingly, China's healthcare service industry is in a golden period of rapid development. In contrast with the development of tourism, thanks to the steady growth in the income of urban and rural residents and the accelerated development of tourism transport infrastructure, such as highways and high-speed railways (40), tourism has also developed to a certain extent, but at a slower pace. Considering this gap, rapid development of health services can influence tourism and cause a range of social problems $(36,37)$. Therefore, paying attention to the balanced development of both aspects at different historical stages is important to improve the level of coupling and coordination and promote the sustainable development of both systems. In addition, the study results are objective and credible and help in extensively understanding the complex relationship between tourism development and medical services, providing a theoretical basis for measuring the coupled coordination of tourism development and medical services in other countries. The findings help governments guide the development of medical tourism practices and implement sustainable development strategies $(15,18)$.

\section{Spatial Dynamic Changes}

In terms of spatial patterns, the coupled coordination values of tourism development and medical services in China are higher in the north provinces than in the south and higher in the east than in the west. However, the coordination between regions has improved to a certain extent, with the north-south direction being more stable and the gap between the east and west being gradually reduced. This change is a clear indication that the Chinese government's development strategy of "promoting the development of the west, the comprehensive revitalization of the northeast, the rise of the central region, and the pioneering development of the east" has achieved significant results (79). The hotspots and sub-hotspots of the coupling of tourism and healthcare services in China are basically distributed along the eastern coast in a strip-like pattern, with a gradient distribution pattern from the coast to the interior (51). However, the CCD of tourism development and medical services in China has varying spatial distribution patterns at different stages. There is a significant spatial dependence of CCD with a clear clustering effect in 2012-2016, and provinces with high (or low) CCD are spatially adjacent to each other (39). The CCD correlation was not significant in 2017-2019, tending to a random distribution with no similarity.

The above results may be related to a number of influencing factors. First, the vast size of China and the large differences in natural conditions and socio-economic development levels among provinces resulted in inconsistencies in the level of development of tourism and healthcare services, which ultimately lead to the two systems being uncoordinated. Therefore, paying attention to inter-regional coordination and reducing regional differences are important. Second, macro policies are important drivers to improve the level of coordination between tourism development and medical services $(44,80)$. Moreover, in medical services, local governments need to take advantage of local conditions and policies to encourage tourism and medical services to go hand in hand. Finally, the relationship between tourism development and medical services can be described as an interaction $(20,21)$. Tourism promotes the development of medical services, medical services support the development of tourism, and the two complement each other in a dynamic process $(9,14,35)$. Therefore, cities that need to vigorously develop medical tourism can enjoy the benefits of coordinated development of tourism development and medical services, avoiding the negative effects of external shocks and maintaining sustainability (53). 


\section{CONCLUSIONS AND IMPLICATIONS}

This work constructs an evaluation index system and uses IDLEM, CCDM, trend surface analysis, and spatial autocorrelation analysis methods in geography to study the relationship of coupling coordination between tourism development and medical services in China from 2012 to 2019. Furthermore, the dynamic change mechanism and spatial distribution of the coupling coordination between tourism development and medical service are explored. The main findings are as follows.

(1) The comprehensive level of tourism development and medical services in China has shown a year-on-year increase, and the two systems are developing in the same way. However, the gap between them is increasing, and medical services are developing faster than tourism development.

(2) The coupling coordination degree shows a good upward trend. However, with only the barely balanced development tourism development lagging behind the stage, the level of coupling coordination needs to be improved. The overall spatial pattern of the coupling coordination degree is "high in the north and low in the south, high in the east and low in the west," with a more obvious trend in the east-west direction.

(3) From a global perspective, the coupling coordination degree in tourism development and medical services has a significant spatial clustering trend, but it gradually evolves into a random distribution pattern over time. In terms of local spatial patterns, an overall spatial development pattern of "cold in the northwest and hot in the southeast" was observed, with a clear pattern of gradient distribution.

(4) The analysis of the driving mechanism of the coupled tourism development and medical services found that the power of the government to regulate is always an important factor influencing the coordination development. Meanwhile, the level of economic development and urbanization also has a strong influence. The other factors have different degrees of influence on the coupling coordination degree in different years.

\section{Implications}

In a theoretical sense, a comprehensive evaluation index system for the coupling coordination of tourism development and medical services in China has been constructed and quantitatively studied. This method can be used to explore the degree of coupling coordination of tourism development and medical services in different countries. This method can be extended to multiple systems, which is conducive to promoting the benign development of multiple systems.

The study findings have practical implications for the promotion of medical tourism in China. First, the Chinese government has attached great importance to the development of medical tourism in recent years, and the results can provide the government with implications for focusing on developing advantageous regions and driving weak regions. Second, the findings can provide a reference for the government to formulate relevant policies to promote the deep integration of medical services and tourism development, fully explore Chinese medicine resources, and transform them into tourism products to optimize investment. Third, enhancing cooperation between healthcare providers and tourism businesses is conducive, especially in the private healthcare services sector. For example, travel agencies work with hospitals or clinics to offer medical and tourism packages to tourists. Hotel providers and herbal medicine companies collaborate to offer a variety of medicinal bath products. Cooperation between healthcare providers and tourism enterprises can build good partnerships and increase profitability and competitiveness.

\section{Limitations}

Although this work has established a comprehensive indicator system to study the relationship of coupled coordination between tourism development and medical services, using the 31 provinces of China as the study area, it has limitations. First, no similar studies have been conducted in other countries on the coupled coordination model of tourism development and medical services proposed, making comparisons on the same evaluation criteria considering the different national contexts of each country difficult. Second, this study starts at the macrolevel of the province, which has implications for grasping the coupled coordinated development of tourism development and medical services in China. If we could start at the micro-level, such as the municipal and county levels, the data would be more accurate, and the conclusions drawn would be more reasonable and scientific. Finally, the research data are derived from existing government statistics, which are not updated on a timely basis and do not allow for timely updating of data to draw the most up-to-date conclusions.

\section{DATA AVAILABILITY STATEMENT}

The original contributions presented in the study are included in the article/supplementary material, further inquiries can be directed to the corresponding author.

\section{AUTHOR CONTRIBUTIONS}

SX: conceptualization, methodology, software, writingoriginal draft, and writing-review and editing. YZ: conceptualization, methodology, and writing-original draft. RL: writing-review and editing and supervision. MZ: supervision, conceptualization, and writing-original draft. JH: software and writing-original draft. GL and JM: conceptualization and writing-original draft. All authors contributed to the article and approved the submitted version. 


\section{REFERENCES}

1. Moghavvemi S, Ormond M, Musa G, Mohamed Isa CR, Thirumoorthi T, Bin Mustapha MZ, et al. Connecting with prospective medical tourists online: a cross-sectional analysis of private hospital websites promoting medical tourism in India, Malaysia and Thailand. Tourism Manage. (2017) 58:15463. doi: 10.1016/j.tourman.2016.10.010

2. Kim S, Arcodia C, Kim I. Critical success factors of medical tourism: the case of south Korea. Int J Environ Res Public Health. (2019) 16:4964. doi: 10.3390/ijerph16244964

3. Pocock NS, Phua KH. Medical tourism and policy implications for health systems: a conceptual framework from a comparative study of Thailand, Singapore and Malaysia. Global Health. (2011) 7:1-12. doi: 10.1186/1744-8603-7-12

4. Lubowiecki-Vikuk A, Dryglas D. Medical tourism services and medical tourism destinations in Central and Eastern Europe-the opinion of Britons and Germans. Econ Res Ekon Istraz. (2019) 32:1256-74. doi: 10.1080/1331677X.2019.1627892

5. Horowitz MD, Rosensweig JA, Jones CA. Medical tourism: globalization of the healthcare marketplace. MedGenMed. (2007) 9:33.

6. Yu JY, Ko TG. A cross-cultural study of perceptions of medical tourism among Chinese, Japanese and Korean tourists in Korea. Tour Manage. (2012) 33:80-88. doi: 10.1016/j.tourman.2011.02.002

7. Garcia-Altes A. The development of health tourism services. Ann Touris Res. (2005) 32:262-6. doi: 10.1016/j.annals.2004.05.007

8. Smith M, Puczkó L. Health and Wellness Tourism. New York, NY: Routledge (2008).

9. Connell J. Contemporary medical tourism: conceptualisation, culture and commodification. Tour Manage. (2013) 34:113. doi: 10.1016/j.tourman.2012.05.009

10. Connell J. Medical tourism: sea, sun, sand and ... surgery. Tour Manage. (2006) 27:1093-100. doi: 10.1016/j.tourman.2005.11.005

11. Taheri B, Chalmers D, Wilson J, Arshed N. Would you really recommend it? Antecedents of word-of-mouth in medical tourism. Tour Manage. (2021) 83:104209. doi: 10.1016/j.tourman.2020.104209

12. Heung VCS, Kucukusta D, Song H. A conceptual model of medical tourism: implications for future research. J Travel Tour Mark. (2010) 27:23651. doi: 10.1080/10548401003744677

13. Tham A. Sand, surgery and stakeholders: a multi-stakeholder involvement model of domestic medical tourism for Australia's Sunshine Coast. Tour Manag Perspect. (2018) 25:29-40. doi: 10.1016/j.tmp.2017.11.002

14. Ganguli S, Ebrahim AH. A qualitative analysis of Singapore's medical tourism competitiveness. Tour Manag Perspect. (2017) 21:74-84. doi: 10.1016/j.tmp.2016.12.002

15. Yan Y. Medical tourism in China: Traditional medicine serving as an emerging tourism resource. In: Luo Y, Jiang J, Bi D, editors. Tourism Product Development in China, Asian and European Countries. Singapore: Springer Singapore (2020). p. 189-97. doi: 10.1007/978-981-15-4447-7_12

16. Heung VCS, Kucukusta D. Wellness tourism in China: resources, development and marketing. Int J Tour Res. (2013) 15:34659. doi: $10.1002 /$ jtr. 1880

17. Fetscherin M, Stephano R-M. The medical tourism index: scale development and validation. Tourism Manage. (2016) 52:539-56. doi: 10.1016/j.tourman.2015.08.010

18. Kazem V. Inbound, outbound, and domestic: The current situation in the chinese medical tourism market. In: Malcolm C, Kazem V, Mayumi H, editors. Current Issues and Emerging Trends in Medical Tourism. Hershey, PA: IGI Global (2015). p. 350-63. doi: 10.4018/978-1-4666-8574-1.ch024

19. Suess C, Baloglu S, Busser JA. Perceived impacts of medical tourism development on community wellbeing. Tourism Manage. (2018) 69:23245. doi: 10.1016/j.tourman.2018.06.006

20. Beladi H, Chao C-C, Ee MS, Hollas D. Does medical tourism promote economic growth? A cross-country analysis. J Travel Res. (2019) 58:12135. doi: 10.1177/0047287517735909

21. Hopkins L, Labonté R, Runnels V, Packer C. Medical tourism today: what is the state of existing knowledge? J Public Health Policy. (2010) 31:18598. doi: 10.1057/jphp.2010.10
22. Ormond M, Sulianti D. More than medical tourism: lessons from Indonesia and Malaysia on South-South intra-regional medical travel. Curr Issues Tour. (2017) 20:94-110. doi: 10.1080/13683500.2014.937324

23. Heung VCS, Kucukusta D, Song H. Medical tourism development in Hong Kong: an assessment of the barriers. Tour Manage. (2011) 32:9951005. doi: 10.1016/j.tourman.2010.08.012

24. Aydin G, Karamehmet B. Factors affecting health tourism and international health-care facility choice. Int J Pharm Healthc Mark. (2017) 11:1636. doi: 10.1108/IJPHM-05-2015-0018

25. Nilashi M, Samad S, Manaf AA, Ahmadi H, Rashid TA, Munshi A, et al. Factors influencing medical tourism adoption in Malaysia: a DEMATEL-Fuzzy TOPSIS approach. Comput Ind Eng. (2019) 137:111. doi: 10.1016/j.cie.2019.106005

26. Rahman Muhammad K. Medical tourism: tourists' perceived services and satisfaction lessons from Malaysian hospitals. Tour Rev. (2019) 74:73958. doi: 10.1108/TR-01-2018-0006

27. Liu L, Chen J. Strategic coupling of urban tourism and regional development in Liaoning Province, China. Asia Pac J Tour Res. (2020) 25:125168. doi: 10.1080/10941665.2020.1851272

28. Epley DR, Menon M. A method of assembling cross-sectional indicators into a community quality of life. Soc Indic Res. (2008) 88:281-96. doi: 10.1007/s11205-007-9190-7

29. Lame E, Eslamitabar S. The ethical \& legal challenges of medicine tourism. J Halal Res. (2020) 4:64-76. doi: 10.31436/iiumlj.v23i2.130

30. Shen X, Qu Y, Wu Q. Assessing the risks of China's medical tourism from the legal perspective. Risk Manag Healthc Policy. (2020) 13:229199. doi: 10.2147/RMHP.S271224

31. Bush M. Addressing the root cause: rising health care costs and social determinants of health. Clin Nutr. (2018) 79:26-29. doi: 10.18043/ncm.79.1.26

32. Hazra NC, Rudisill C, Gulliford MC. Determinants of health care costs in the senior elderly: age, comorbidity, impairment, or proximity to death? Eur J Health Econ. (2018) 19:831-42. doi: 10.1007/s10198-017-0926-2

33. Mahendradhata Y. Proceed with caution: potential challenges and risks of developing healthcare tourism in Indonesia. Global Health. (2019) 14:34050. doi: 10.1080/17441692.2018.1504224

34. De Arellano ABR. Patients without borders: the emergence of medical tourism. Int $J$ Health Serv. (2007) 37:19398. doi: 10.2190/4857-468G-2325-47UU

35. Zarei A, Maleki F. Asian medical marketing, a review of factors affecting Asian medical tourism development. J Qual Assur Hosp Tour. (2019) 20:115. doi: 10.1080/1528008X.2018.1438959

36. Hanefeld J, Smith R, Horsfall D, Lunt N. What do we know about medical tourism? A review of the literature with discussion of its implications for the UK National Health Service as an example of a public health care system. $J$ Travel Med. (2014) 21:410-17. doi: 10.1111/jtm.12147

37. Freire NA. The emergent medical tourism: advantages and disadvantages of the medical treatments abroad. Int Bus Res. (2012) 5:41-50. doi: 10.5539/ibr.v5n2p41

38. Fan Y, Fang C, Zhang Q. Coupling coordinated development between social economy and ecological environment in Chinese provincial capital cities-assessment and policy implications. J Cleaner Prod. (2019) 229:28998. doi: 10.1016/j.jclepro.2019.05.027

39. Zhang Z, Li Y. Coupling coordination and spatiotemporal dynamic evolution between urbanization and geological hazards-a case study from China. Sci Total Environ. (2020) 728:1-12. doi: 10.1016/j.scitotenv.2020.138825

40. Wang Q, Mao Z, Xian L, Liang Z. A study on the coupling coordination between tourism and the low-carbon city. Asia Pac J Tour Res. (2019) 24:550-62. doi: 10.1080/10941665.2019.16 10002

41. Song Q, Zhou N, Liu T, Siehr SA, Qi Y. Investigation of a "coupling model" of coordination between low-carbon development and urbanization in China. Energy Policy. (2018) 121:346-54. doi: 10.1016/j.enpol.2018.05.037

42. Yang C, Zeng W, Yang X. Coupling coordination evaluation and sustainable development pattern of geo-ecological environment and urbanization in Chongqing municipality, China. Sust Cities Soc. (2020) 61:102271. doi: 10.1016/j.scs.2020.1 02271 
43. Tang Z. An integrated approach to evaluating the coupling coordination between tourism and the environment. Tourism Manage. (2015) 46:1119. doi: 10.1016/j.tourman.2014.06.001

44. Cheng L, Zhang J. Assessment of coupling coordination between tourism development and economic growth after the 2008 Wenchuan earthquake: Beichuan, China. Asia Pac J Tour Res. (2020) 25:602-19. doi: 10.1080/10941665.2020.17 52753

45. Lai Z, Ge D, Xia H, Yue Y, Wang Z. Coupling coordination between environment, economy and tourism: a case study of China. PLoS ONE. (2020) 15:e0228426. doi: 10.1371/journal.pone.02 28426

46. Zhou Z, Yang Q, Kim D-J. An empirical study on coupling coordination between the cultural industry and tourism industry in ethnic minority areas. J Open Innov Technol. Mark Complex. (2020) 6:1-15. doi: 10.3390/joitmc60 30065

47. Ding L, Zhao W, Huang Y, Cheng S, Liu C. Research on the coupling coordination relationship between urbanization and the air environment: a case study of the area of Wuhan. Atmosphere. (2015) 6:1539-58. doi: 10.3390/atmos61 01539

48. Liu W, Jiao F, Ren L, Xu X, Wang J, Wang X. Coupling coordination relationship between urbanization and atmospheric environment security in Jinan City. J Cleaner Prod. (2018) 204:1-11. doi: 10.1016/j.jclepro.2018.08.244

49. Shi T, Yang S, Zhang W, Zhou Q. Coupling coordination degree measurement and spatiotemporal heterogeneity between economic development and ecological environment --empirical evidence from tropical and subtropical regions of China. $J$ Cleaner Prod. (2020) 244:118739. doi: 10.1016/j.jclepro.2019.1 18739

50. Guan D, Gao W, Su W, Li H, Hokao K. Modeling and dynamic assessment of urban economy-resource-environment system with a coupled system dynamics-geographic information system model. Ecol Indic. (2011) 11:133344. doi: 10.1016/j.ecolind.2011.02.007

51. Zhu Y, Zhu Q, Zhu Z. Modeling, evaluation and analysis of tourism destination competitiveness: a case study of the Yangtze river Delta of China. Asia Pac J Tour Res. (2014) 19:932-49. doi: 10.1080/10941665.2013.8 33122

52. Yuan Y, Jin M, Ren J, Hu M, Ren P. The dynamic coordinated development of a regional environment-tourism-economy system: a case study from western Hunan province, China. Sustainability. (2014) 6:5231-51. doi: 10.3390/su60 85231

53. Liao K-C, Yue M-Y, Sun S-W, Xue H-B, Liu W, Tsai S-B, et al. An evaluation of coupling coordination between tourism and finance. Sustainability. (2018) 10:1-23. doi: 10.3390/su100 72320

54. Zhang $\mathrm{H}, \mathrm{Gu} \mathrm{C}-\mathrm{l}, \mathrm{Gu} \mathrm{L}-\mathrm{w}$, Zhang Y. The evaluation of tourism destination competitiveness by TOPSIS \& information entropy - a case in the Yangtze River Delta of China. Tour Manage. (2011) 32:44351. doi: 10.1016/j.tourman.2010.02.007

55. Maloletko A, Kaurova O, Kryukova E, Pochinok N, Gladko E. Analysis of key indicators of tourism industry in Russia. Modern Appl Sci. (2015) 9:25-33. doi: 10.5539/mas.v9n3p25

56. Huang J-H, Peng K-H. Fuzzy Rasch model in TOPSIS: a new approach for generating fuzzy numbers to assess the competitiveness of the tourism industries in Asian countries. Tour Manage. (2012) 33:45665. doi: 10.1016/j.tourman.2011.05.006

57. Torres-Delgado A, López Palomeque F. The ISOST index: a tool for studying sustainable tourism. J Destin Mark Manag. (2018) 8:28189. doi: 10.1016/j.jdmm.2017.05.005

58. Kong X, Yang Y, Gao J, Guan J, Liu Y, Wang R, et al. Overview of the health care system in Hong Kong and its referential significance to mainland China. J Chin Med Assoc. (2015) 78:569-73. doi: 10.1016/j.jcma.2015.02.006

59. Veselova ES. Medical tourism. Prob Econ Trans. (2017) 59:480500. doi: 10.1080/10611991.2017.1352363

60. Li Z, Zhang W, Kong A, Ding Z, Wei H, Guo Y. Configuration analysis of influencing factors of technical efficiency based on DEA and fsQCA: evidence from China's medical and health institutions. Risk Manag Healthc Policy. (2021) 14:49-65. doi: 10.2147/RMHP.S282178

61. Wen J, Wang CC, Kozak M. Post-COVID-19 chinese domestic tourism market recovery: potential influence of traditional chinese medicine on tourist behaviour. Anatolia. (2021) 32:121-25. doi: 10.1080/13032917.2020.1768335

62. Wang W-Y, Zhou H, Wang Y-F, Sang B-S, Liu L. Current policies and measures on the development of traditional Chinese medicine in China. Pharmacol Res. (2021) 163:1-11. doi: 10.1016/j.phrs.2020.105187

63. Fisher CL, Ledford CJ, Moss DA, Crawford P. Physician communication to enhance patient acupuncture engagement in family medicine. $J$ Health Commun. (2018) 23:422-29. doi: 10.1080/10810730.2018.1458924

64. Ridderstaat J, Singh D, DeMicco F. The impact of major tourist markets on health tourism spending in the United States. J Destin Mark Manag. (2019) 11:270-80. doi: 10.1016/j.jdmm.2018.05.003

65. Wu H, Hao Y, Weng J-H. How does energy consumption affect China's urbanization? New evidence from dynamic threshold panel models. Energy Policy. (2019) 127:24-38. doi: 10.1016/j.enpol.2018.11.057

66. Zhao J, Tang J. Industrial structure change and economic growth: a China-Russia comparison. China Econ. Rev. (2018) 47:219-33. doi: 10.1016/j.chieco.2017.08.008

67. Li B, Chen C, Hu B. Governing urbanization and the new urbanization plan in China. Environ Urban. (2016) 28:515-34. doi: 10.1177/0956247816647345

68. Zameer $\mathrm{H}$, Yasmeen $\mathrm{H}$, Wang $\mathrm{R}$, Tao J, Malik MN. An empirical investigation of the coordinated development of natural resources, financial development and ecological efficiency in China. Resour Pol. (2020) 65:101580. doi: 10.1016/j.resourpol.2020.101580

69. Li Y, Li Y, Zhou Y, Shi Y, Zhu X. Investigation of a coupling model of coordination between urbanization and the environment. J Environ Manage. (2012) 98:127-33. doi: 10.1016/j.jenvman.2011.12.025

70. Fu S, Zhuo H, Song H, Wang J, Ren L. Examination of a coupling coordination relationship between urbanization and the eco-environment: a case study in Qingdao, China. Environ Sci Pollut Res Int. (2020) 27:23981-93. doi: 10.1007/s11356-020-0 8683-7

71. Hu M, Li Z, Yuan M, Fan C, Xia B. Spatial differentiation of ecological security and differentiated management of ecological conservation in the Pearl River Delta, China. Ecol Indic. (2019) 104:439-48. doi: 10.1016/j.ecolind.2019.04.081

72. Yanbo Q, Guanghui J, Yuting Y, Qiuyue Z, Yuling L, Wenqiu M. Multi-scale analysis on spatial morphology differentiation and formation mechanism of rural residential land: a case study in Shandong Province, China. Habitat Int. (2018) 71:135-46. doi: 10.1016/j.habitatint.2017.11.011

73. Ord JK, Getis A. Testing for local spatial autocorrelation in the presence of global autocorrelation. J Reg Sci. (2001) 41:411-32. doi: 10.1111/0022-4146.00224

74. Liu Y, Yang L, Jiang W. Coupling coordination and spatiotemporal dynamic evolution between social economy and water environmental quality - a case study from Nansi Lake catchment, China. Ecol Indic. (2020) 119:1-12. doi: 10.1016/j.ecolind.2020.1 06870

75. Ruan W, Li Y, Zhang S, Liu C-H. Evaluation and drive mechanism of tourism ecological security based on the DPSIR-DEA model. Tour Manage. (2019) 75:609-25. doi: 10.1016/j.tourman.2019.06.021

76. Zuo Y, Chen H, Pan J, Si Y, Law R, Zhang M. Spatial distribution pattern and influencing factors of sports tourism resources in China. ISPRS Int J Geo-Inf. (2021) 10:428. doi: 10.3390/ijgi100 70428

77. Cheniti H, Cheniti M, Brahamia K. Use of GIS and Moran's I to support residential solid waste recycling in the city of Annaba, Algeria. Environ Sci Pollut Res Int. (2020) 27:1-15. doi: 10.1007/s11356-020-1 0911-z

78. Xu S, Zuo Y, Zhang M. Evaluation of tourism ecological security and diagnosis of obstacle factors in china based on fuzzy object element model. Sci Geogr Sin. (2021) 41:33-43. doi: 10.13249/j.cnki.sgs.2021.01.004

79. Li J, Li M, Gao Y, Li J, Su H, Huang M. Regional level: New landscape of competition and cooperation among regions in China. In: Li J, Li M, Gao Y, Li J, Su H, Huang M, editors. China's Provincial Economic Competitiveness 
and Policy Outlook for the 13th Five-year Plan Period (2016-2020). Singapore: Springer Singapore (2018). p. 135-62. doi: 10.1007/978-981-13-2664-6_4

80. Suhel S, Bashir A. The role of tourism toward economic growth in the local economy. Econ J Emerg Mark. (2018) 10:32-39. doi: 10.20885/ejem.vol10.iss1.art4

Conflict of Interest: The authors declare that the research was conducted in the absence of any commercial or financial relationships that could be construed as a potential conflict of interest.

Publisher's Note: All claims expressed in this article are solely those of the authors and do not necessarily represent those of their affiliated organizations, or those of the publisher, the editors and the reviewers Any product that may be evaluated in this article, or claim that may be made by its manufacturer, is not guaranteed or endorsed by the publisher.

Copyright (c) $2022 \mathrm{Xu}, \mathrm{Zuo}$, Law, Zhang, Han, Li and Meng. This is an open-access article distributed under the terms of the Creative Commons Attribution License (CC $B Y)$. The use, distribution or reproduction in other forums is permitted, provided the original author(s) and the copyright owner(s) are credited and that the original publication in this journal is cited, in accordance with accepted academic practice. No use, distribution or reproduction is permitted which does not comply with these terms. 